\title{
Systemic Thrombolysis for Intraoperative, High Risk Pulmonary Embolism. Clinical Case
}

\author{
Anna Grzanka, Pilar Reboto, Alfredo Plaza, Patricia Gaite \\ Anesthesiology and Reanimation Department, University Trust Hospital (Hospital Universitario Fundación), Alcorcón, Spain \\ Email: grzanka24@gmail.com
}

How to cite this paper: Grzanka, A., Reboto, P., Plaza, A. and Gaite, P. (2019) Systemic Thrombolysis for Intraoperative, High Risk Pulmonary Embolism. Clinical Case. Open Access Library Journal, 6: e5411. https://doi.org/10.4236/oalib.1105411

Received: April 18, 2019

Accepted: July 7, 2019

Published: July 10, 2019

Copyright $\odot 2019$ by author(s) and Open Access Library Inc.

This work is licensed under the Creative Commons Attribution International License (CC BY 4.0).

http://creativecommons.org/licenses/by/4.0/ (c) (i) Open Access

\begin{abstract}
A 68-year-old woman with intercondylar fracture of the femur suffers massive (high risk) pulmonary embolism (PE) during surgery and is successfully treated with systemic thrombolysis. After limb exsanguinations with Esmarch bandage, the patient presents with sudden oxygen desaturation (99\% to $80 \%$ ) with subsequent dyspnea and hypotension. She is intubated and requires continuous adrenalin perfusion while the surgery is finished and until she can be transferred to the reanimation ward. We decide to perform transthoracic echocardiogram (her general condition impedes transfer to tomography) which confirms right ventricular overload and pulmonary hypertension. These findings justify systemic thrombolysis, which is performed with good results. Surgery, and in particular orthopedic surgery, increase the risk of PE. When considering high risk PE, guidelines recommend primary reperfusion strategy through systemic thrombolysis (which can be contraindicated in surgery patients) or catheter-assisted thrombus removal (less widely available). Lately, surgical pulmonary embolectomy is being discussed as a treatment option for patients with contraindication to thrombolysis, but this practice is still uncommon.
\end{abstract}

\section{Subject Areas}

Anaesthesiology \& Pain Management

\section{Keywords}

Anesthesiology, Femur Fracture, Orthopedic Surgery, Thrombolysis, High Risk Pulmonary Embolism

\section{Introduction}

We analyze our case with reference to 2014 ESC Guidelines on the diagnosis and 
management of acute pulmonary embolism, UpToDate- "Treatment, prognosis, and follow-up of acute pulmonary embolism in adults" (update Jul, 2018) and "Thrombolytic (fibrinolytic) therapy in acute pulmonary embolism and lower extremity deep vein thrombosis" (update Jan, 2018) and an article from Advances in Anesthesia (2017)—“Update on Perioperative Pulmonary Embolism Management".

The patient has provided written consent to publish this case report.

\section{Information about the Patient}

A 68-year-old women. Non-smoker. Possible allergy to seafood.

Personal history of endometrial cancer stage IA treated with surgery in combination with chemotherapy in 2012 (completed oncology revisions in 2017). Surgical history of open reduction and internal fixation of left olecranon fracture (April 2017). No chronic treatment. NSAIDs occasionally for ankle pain. No family or personal history of thrombophilia.

\section{Clinical Findings}

She is admitted to the hospital with intercondylar fracture of the left femur and the operation takes place three days later. Antithrombotic prophylaxis with bemipar in $3500 \mathrm{UI}$ a day is administered only on the day of admission because of possible surgery.

Intraoperative: Spinal anesthesia and sedation are performed. After limb exsanguinations with Esmarch bandage the patient presents with sudden oxygen desaturation (99\% to $80 \%$ ) which is initially managed as oversedation but fails to improve. Lungs were clear to auscultation and she did not present dyspnea. Tranexamic acid perfusion was stopped. (after administration of about $200 \mathrm{mg}$ ). Radial artery is cannulated and blood gas with $\mathrm{FiO}_{2} 0.5$ shows severe hypoxemia. $\left(51 \mathrm{PO}_{2}, 31 \mathrm{PCO}_{2}, \mathrm{pH} 7.42, \mathrm{HCO}_{3}^{-}\right.$19, EB -3.6). The patient continues to exhibit desaturation (75\%) followed by dyspnea and finally requires intubation. There is no improvement in saturation even with $\mathrm{FiO}_{2} 1$. Exhaled carbon dioxide marks $21 \mathrm{mmHg}$ compared to $31 \mathrm{mmHg}$ in blood gas. After short period of hypertension, she presents with tachycardia and hypotension $(60 / 30 \mathrm{mmHg})$ which requires adrenalin perfusion (up to $0.9 \mathrm{mcg} / \mathrm{kg} / \mathrm{min}$ ). While performing supportive procedures, the surgery is finished (around 60 minutes). With suspicion of high risk pulmonary embolism (which definition is shown in Figure 1), the patient is transferred to the reanimation unit, depending on mechanical ventilation and with vasoactive drug perfusion.

\section{Diagnosis}

Once in the reanimation ward, the patient is still hemodynamically unstable, so we reject transferring her to tomography (following 2014 ESC Guidelines as on Figure 2). We decide instead to perform echocardiography, which shows signs compatible with pulmonary embolism. 


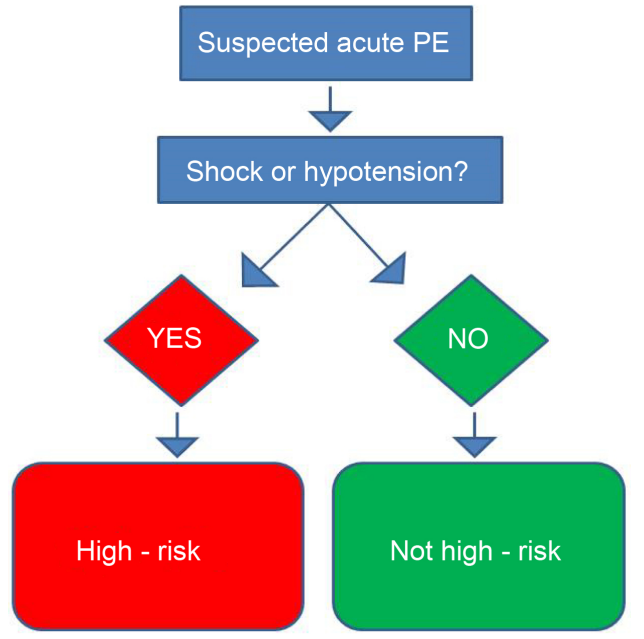

Figure 1. PE is termed high-risk when accompanied by shock or hypotension (systolic blood pressure $<90 \mathrm{mmHg}$ ). Adapted from 2014 ESC Guidelines on the diagnosis and management of acute pulmonary embolism [1].

\section{Suspected high - risk PE}

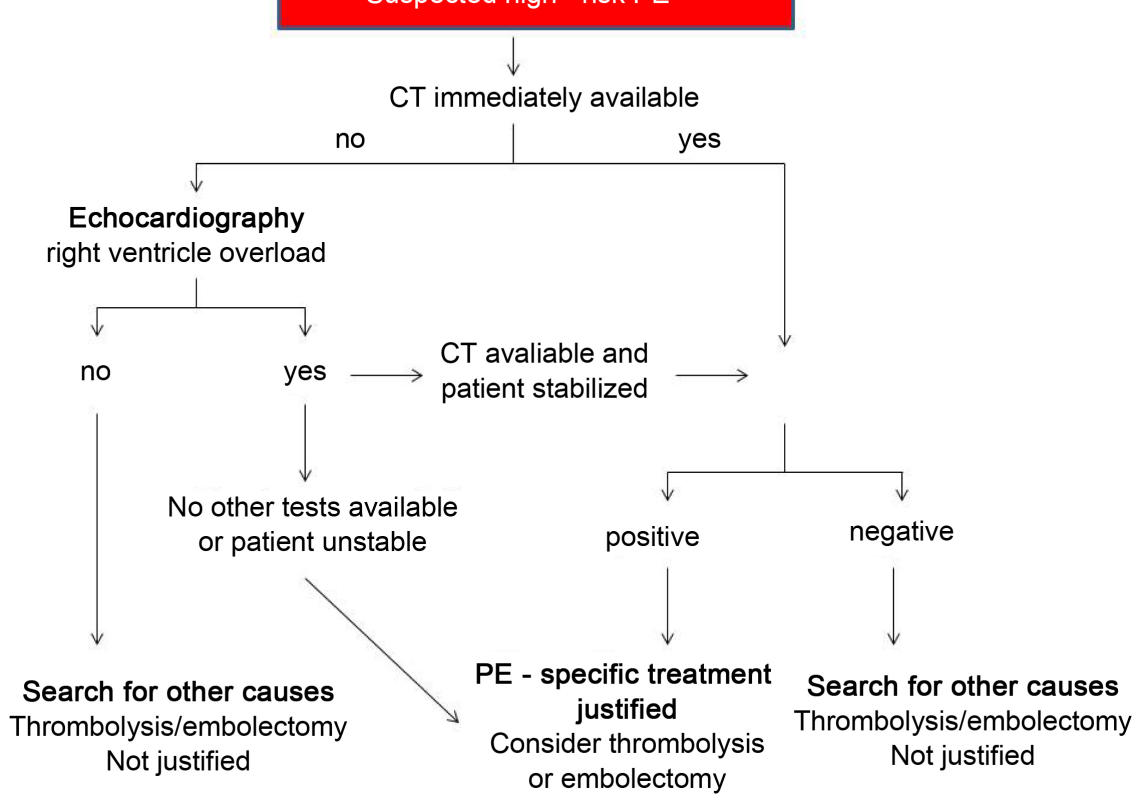

Figure 2. In high-risk PE suspicion and when CT is not available or transfer is impossible echocardiography is recommended. It can justify more aggresive treatment but cannot confirm diagnosis. Adapted from 2014 ESC Guidelines on the diagnosis and management of acute pulmonary embolism [1].

Translated: Severe dilatation of right ventricle with TAPSE $17 \mathrm{~mm}$ but with dysfunction of free wall.

Moderate-to-severe tricuspid insufficiency.PSP of $46+$ dilated vena cava $=66$ mmHg. Leftward motion of the interventricular septum. Left ventricle not dilated with normal ejection fraction. 


\section{Treatment}

Echocardiographic signs of right ventricle overload and hemodynamic instability justify primary reperfusion therapy (Figure 2). The only available option in our center is systemic thrombolysis. Although our patient underwent surgery less than an hour earlier, we decide to proceed with the treatment in view of the general worsening in her condition. Through central venous catheter we administer $100 \mathrm{mg}$ of Recombinant Tissue Plasminogen Activator (rt-PA) over a period of two hours followed by sodium heparin perfusion. She still depends on vasoactive drugs, which are changed from adrenalin to noradrenalin $(0.05-0.8$ $\mathrm{mcg} / \mathrm{kg} / \mathrm{min}$ ). Twenty hours later her condition improves enough to perform tomography, which confirms the presence of pulmonary embolism as shown on Figure 3.

\section{Translated:}

Computer tomography with intravenous contrast.

Confirms intraluminal filling defect compatible with $P E$ affecting the right hemithorax. The embolus occludes the principal pulmonary artery, upper lobar artery and intermediate artery. It extends to the medial lobar and segmental branches in the basal pyramid and upper lobe. The left hemithorax presents filling defect in the lingular artery and segmental branches of the upper left lobe and basal pyramid. Bibasilar subpleural atelectasis and small apical infarction are observed in the left lung. Right heart chambes dilatation and flattening of the interventricular septum are detected as signs of right ventricle overload. Small bilateral pleural effusion.

Conclusion: Findings compatible with massive pulmonary embolism with signs of right ventricle overload.

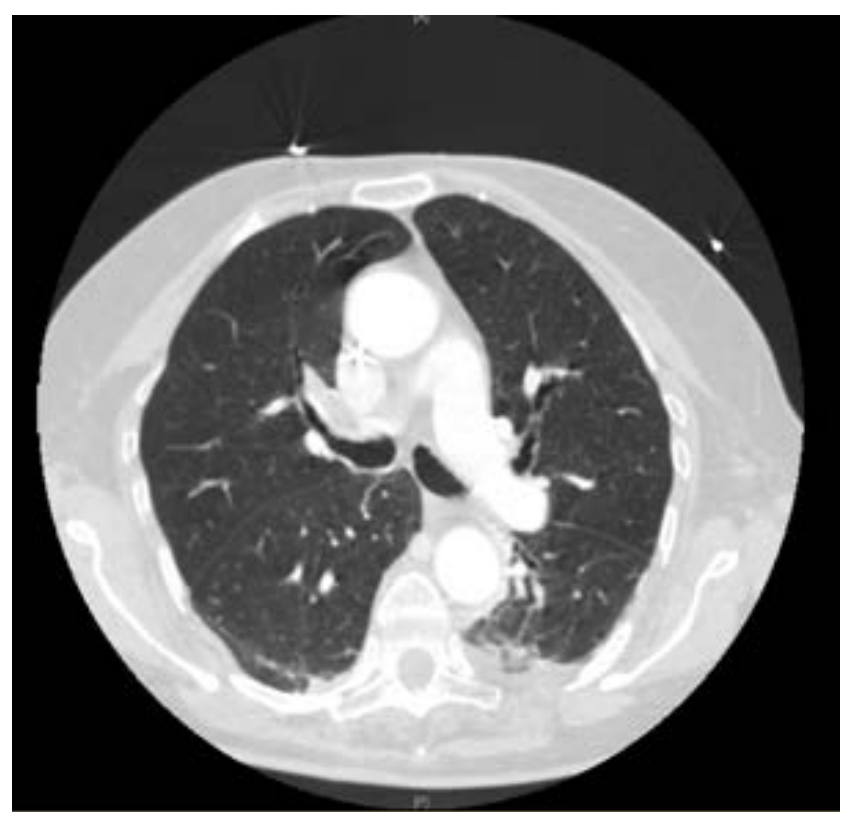

Figure 3. Computer tomography scan with thrombus in right pulmonary artery. 
On the second day after surgery we change anticoagulation from sodium heparin perfusion to low molecular weight heparin (enoxaparin $40 \mathrm{mg}$ every 12 hours). On the third day the patient is extubated and vasoactive drugs are withdrawn and on the fifth day she is transferred to the orthopedic ward. No signs of bleeding are observed during her stay. Nevertheless one red blood cell concentrate is transfused.

\section{Results}

On the orthopedic ward the patient progresses well and continues with no signs of bleeding. Venous Doppler ultrasound of the lower limbs is performed and confirms deep vein thrombosis in external gastrocnemius veins of the left leg. Echocardiography is repeated as below:

Translation: Left ventricle not dilated with normal wall thickness and good systolic function. Impaired diastolic function. Normal pressure. Slightly enlarged right ventricle with TAPSE $13 \mathrm{mmHg}$ and hypocinesia of the free wall. Mild tricuspid insufficiency with PSAP slightly increased. DSVD present.

The patient is discharged on the twelfth day after surgery with oral anticoagulation (apixaban $5 \mathrm{mg}$ every 12 hours).

Three months later, thoracic tomography is repeated showing complete resolution of pulmonary embolism.

\section{Discussion}

Pulmonary embolism is termed massive in $8 \%$ of cases with a $30 \%-50 \%$ mortality rate [4]. This explains the importance of effective diagnosis and management. The more striking the symptoms and the more serious the thromboembolism, the more evident the clinical indicators and the more complex the therapeutic decision-making and vice versa. We have been unable to find and there probably does not exist any guidelines for intraoperative PE. In 2017 in Advances in Anaesthesia, Smeltz, Kolarczyk and Isaak published an update on the management of perioperative PE. Their recommendations are resumed in Figure 4 (adapted from the article).

Should we anticoagulate earlier?

According to ESC guidelines unfractionated heparin should be administered as soon as high risk PE is suspected. However, this is less clear in the case of high bleeding risk patients e.g. surgical patients. According to Smeltz, Kolarczyk and Isaak, major bleeding should be controlled before anticoagulation (Figure 4) [2]. Should we treat all intraoperative PE with catheter based techniques?

We have to take into account the risk of bleeding (surgery type, general condition of the patient, etc.), available hospital equipment and our experience. Not all surgical procedures contraindicate thrombolysis and if the patient remains unstable, there is no absolute contraindication to performing this treatment. On the one hand, transcutaneous procedures carry a lower bleeding risk but on the other hand, they are not widely available in all hospitals. Systemic 


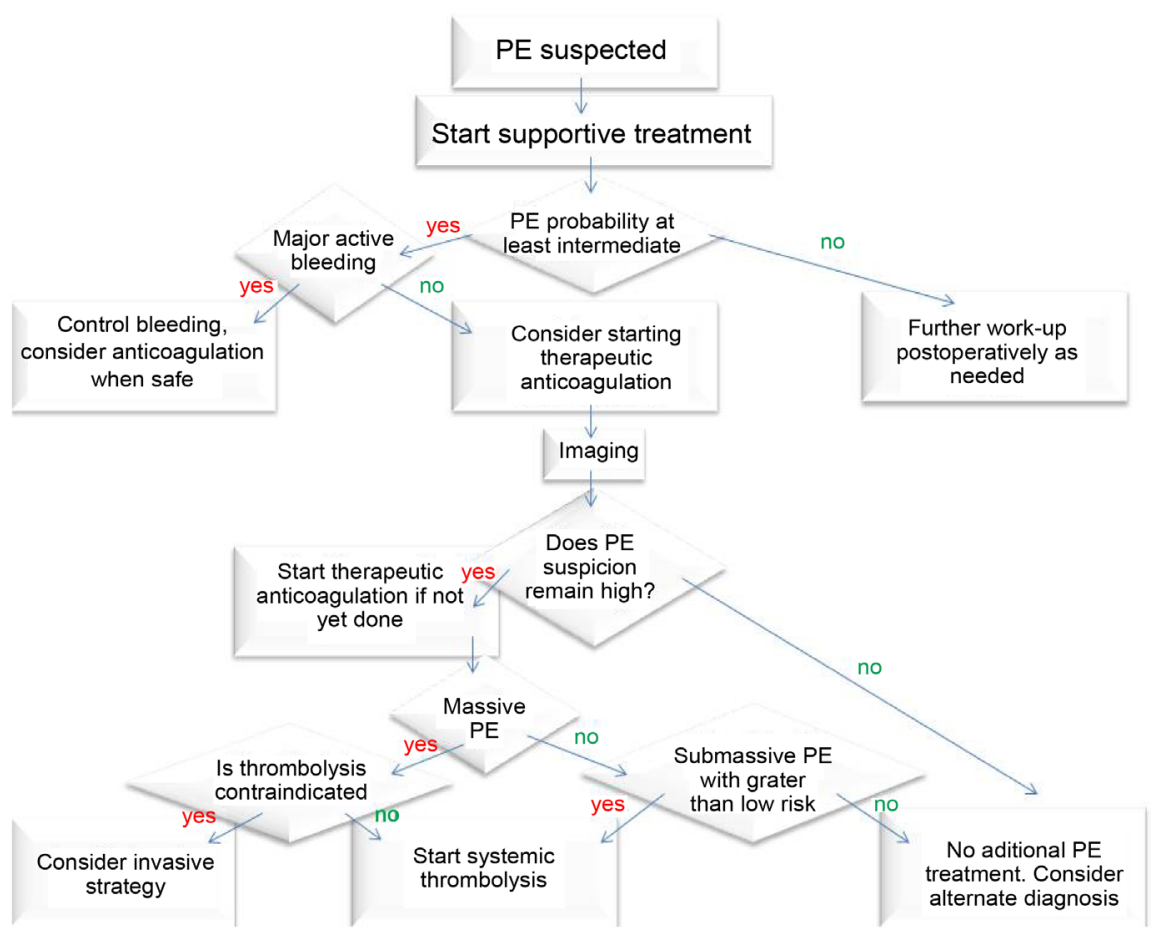

Figure 4. Management of intraoperativa PE suspicion. Adapted from Update on Perioperative Pulmonary Embolism Management Alan M. Smeltz MD, Lavinia M. Kolarczyk MD y Robert S. Isaak DO [2].

thrombolysis remains the fastest treatment option in the majority of centers [3].

What would we do in the case of high bleeding risk surgery?

In the absence of a catheter-based treatment and where the patient's general condition does not allow transfer to another center, we only have two options: either systemic thrombolysis with the corresponding risk of bleeding, or anticoagulation alone with the risk of not being sufficiently effective. In the absence of guidelines, both options are valid, and an evaluation of the patient's condition and response to supportive procedures will determine the choice [1] [2].

Why rt-PA?

Although rt-PA is the most commonly used thrombolytic, the superiority of any one agent or regimen over another has not been established. In the United States, since the approval of the two-hour administration regime, only rt-PA is in use. It seems that streptokinase has the highest potential to trigger allergic reaction [4].

Clearly prevention is better than cure. The American Academy of Orthopaedic Surgeons suggested in 2014 that operation on a hip fracture within the first 48 hours is associated with better outcomes. It is probable that the longer the patient waits, the higher is the risk of complications [5].

\section{Conflicts of Interest}

The authors declare no conflicts of interest regarding the publication of this paper. 


\section{References}

[1] Konstantinides, S.V., Torbicki, A., Agnelli, G., Danchin, N., Fitzmaurice, D., Galie` N., Gibbs, J.M.R., Huisman, M.V., Humbert, M., Kucher, N., Lang, I., Lankei, M., Lekakis, J., Maack, C., Mayer, E., Meneveau, N., Perrier, A., Pruszczyk, P., Rasmussen, L.H., Schindler, T.H., Svitil, P. and Noordegraaf, A.V. (2014) 2014 ESC Guidelines on the Diagnosis and Management of Acute Pulmonary Embolism: The Task Force for the Diagnosis and Management of Acute Pulmonary Embolism of the European Society of Cardiology (ESC) Endorsed by the European Respiratory Society (ERS). European Heart Journal, 36, 2642.

https://doi.org/10.1093/eurheartj/ehu479

[2] Smeltz, A.M., Kolarczyk. L.M. and Isaak. R.S. (2017) Update on Perioperative Pulmonary Embolism Management: A Decision Support Tool to Aid in Diagnosis and Treatment. Advances in Anesthesia, 35, 213-228. https://doi.org/10.1016/j.aan.2017.08.001

[3] Tapson, V.F., Weinberg, A.S., Phil, M., Mandel, J. and Finlay, G. (2018) Thrombolytic (Fibrinolytic) Therapy in Acute Pulmonary Embolism and Lower Extremity Deep Vein Thrombosis.

https://www-uptodate-com.m-hufa.a17.csinet.es/contents/thrombolytic-fibrinolytic -therapy-in-acute-pulmonary-embolism-and-lower-extremity-deep-vein-thrombosi s? search=Thrombolytic\%20therapy\%20in\%20acute\%20pulmonary\%20embolism\&s ource $=$ search result\&selectedTitle $=1 \sim 150 \&$ usage type $=$ default $\&$ display $r a n k=1$

[4] Tapson, V.F., Weinberg, A.S., Phil, M., Mandel, J., Hockberger, R.S., FACEP and Finlay, G. (2018) Treatment, Prognosis, and Follow-Up of Acute Pulmonary Embolism in Adults.

https://www-uptodate-com.m-hufa.a17.csinet.es/contents/treatment-prognosis-and -follow-up-of-acute-pulmonary-embolism-in-adults?search=Treatment $\% 20$ prognos is $\% 20$ and $\% 20$ follow $\% 20$ up $\% 20$ pulmonary $\% 20 \mathrm{embolism \& source=search} \mathrm{result \& sel}$ ectedTitle $=1 \sim 150 \&$ usage type $=$ default \&display $\operatorname{rank}=1$

[5] American Academy of Orthopaedic Surgeons-Clinical Practice Guidelines. http://www.orthoguidelines.org/guideline-detail?id=1233 